Behavioural Sciences | Jan Scheffel

\title{
On the solvability of the mind-body problem
}

The mind-body problem is one of the most enigmatic issues in philosophy that has yet to be resolved. Professor Jan Scheffel from KTH Roya Institute of Technology, Sweden analyses the mind-body problem from a finds that consciousness is epistemologically emergent epistemologically emergen
and shows that this result overlaps with the problem of free will. If a theory for consciousness could be constructed, free will would not exist. Professor Scheffel discloses that the mind-bod problem cannot be solved the notion of emergence in an argument for free will.
$\mathrm{T}$ fundamental issue in philosophy There is an expanse of literature concerning the mind-body problem dating back to Descartes and beyo and the problem has challenged many other philosophers over several
thousand years. There exist somewhat different interpretate exist somewh how the mind-body prom should be formulated Broadly speaking one may say that it involves the questions may say that it involves the questions by the physical and, in that case, how the functions of the mind, in particul consciousness, can be understood from the physical. This open question is one of the most enigmatic issues in philosophy that has yet to be resolved.

Jan Scheffel, Professor in Theoretical Fusion Plasma Physics at the School of Electrical Engineering and Computer Science (EECS), KTH Roya Institute of Technology, Sweden from a physicalist perspective, that is the philosophical standpoint that 'everything is physical' and all

phe PHYSICALISM

It is relatively common amon contemporary philosophers of mind to non-reductive physictive physicalist or chiming that the mind is not separate from the body. These practices are prominent in scientific fields, particularly within the various neurosciences, computer science (particularly artificial intelligence), sociobiology and evolutionary psychology.

Reductive physicalists profess that all mental states and properties will ultimately

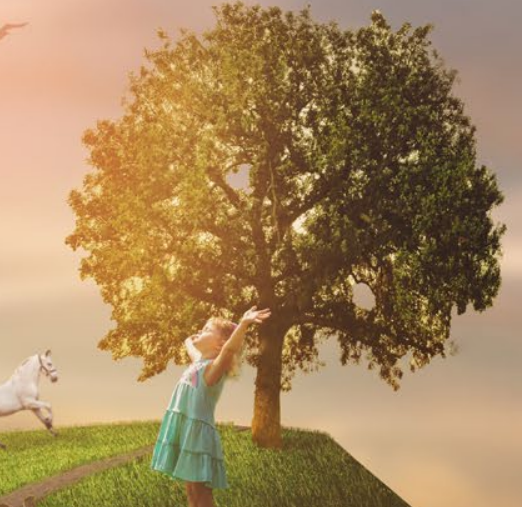

be explained by scientific accounts of physiological processes and states. contrast, non reductive physicalists cannot explain consciousness. While we normally seek scientific understanding from a reductionist perspective, analysing and describing complex phenomena in terms of their fundamental constituents, it is difficult to apply this methodology to consciousness, which has naturally evolved over millions of years into a multifaced system with complex highlevel properties.

\section{EMERGENCE}

This research combines the concep of emergence with findings from gurithmic information theory and quantum mechanics. Emergence system is observed to possess properties that its parts do not have individually These properties, or behaviours, only arise when the parts interact making up the whole. Emergence occurs in complex systems with attributes that are difficult or impossible to reduce to the parts of the systems. Emergent phenomena can be epistemological, in that it relates to the theory of knowledge, or ontological, relating to existence. Epistemologically emergent phenomena cannot be understood from theories for their associated low-level constituents. Ontologically energent phenomena their lownot even be pedsced to their low level constiuents, one could to nature.

\section{JUMPING ROBOT}

\section{THOUGHT EXPERIMENT}

In order to comp can occur in a system, Professor Scheffe devised a thought experiment involving a jumping robot. It is envisaged that a group of robots are located on a remote island. The robots are of a commo design and they are programmed to walk freely around the island and perform particular tasks. The robots are instructed to carry out their duties as effectively as possible and they can communicate with each other. When a robot finds a particular action that teaches it to the other robots.

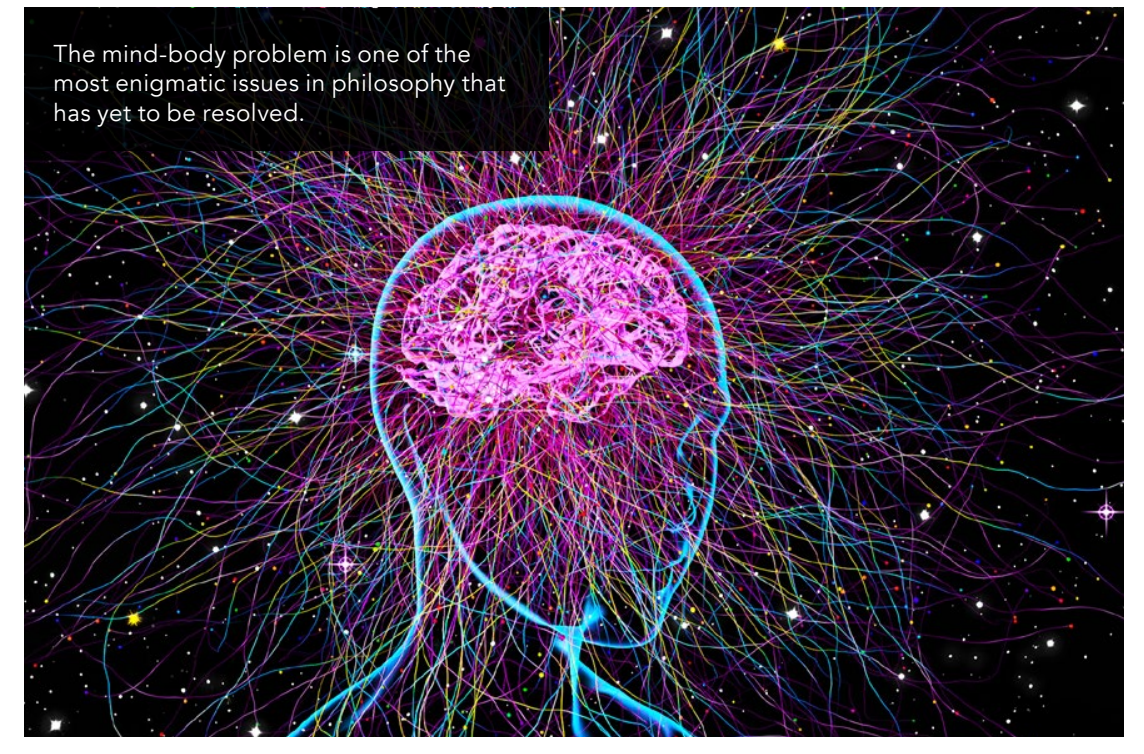

Professor Scheffel created a (l) discrete logistc equation to simulate jump. The task was aban a controlled the robots' movents. It is assumed robots remained on the island for some of these valuable actions. Although jumping is a familiar action, and robots exist that can jump, this particular

time unobserved.

On returning to the island it is observed that some of the robots

Professor Scheffel combines the concept of emergence with his findings from algorithmic information theory and quantum mechanics.

robot's limbs, joints and muscles could not be coordinated to produce stable jumping behaviour. Due to the motion, the model fails to come up with

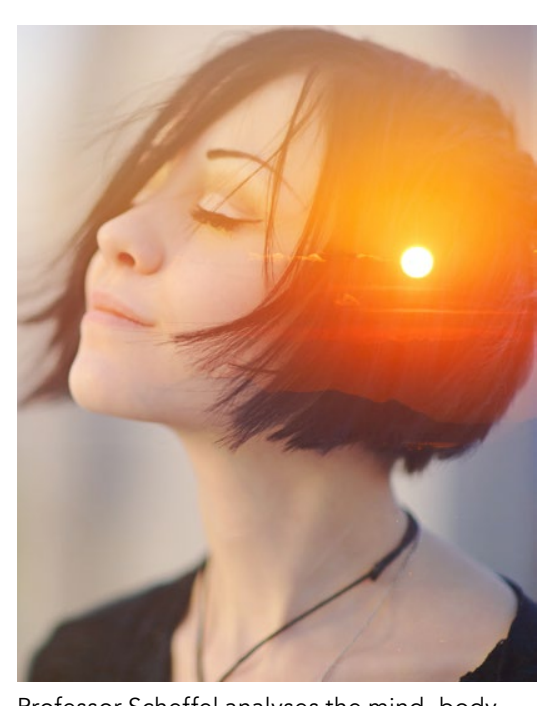

can jump over obstacles. There is no explanation or theory as to how the thoir ann devices, a robot was ablo their own its ess, a robot was able to robots' ability to jump evolved. This property of being able to jump is an

CONSCIOUSNESS

EPISTEMOLOGICALLY EMERGENT

This thought experiment serves

as an analogy of the neurological functions relating to consciousness, Consciousness is well known to us to supervene on its low-level basis of neurons. Epistemologically, however, an overall theory for consciousness based on neural behaviour cannot be formulated. Professor Scheffel describes this explanatory gap between individual neuronal activity and consciousness
as a result of that consciousness is 


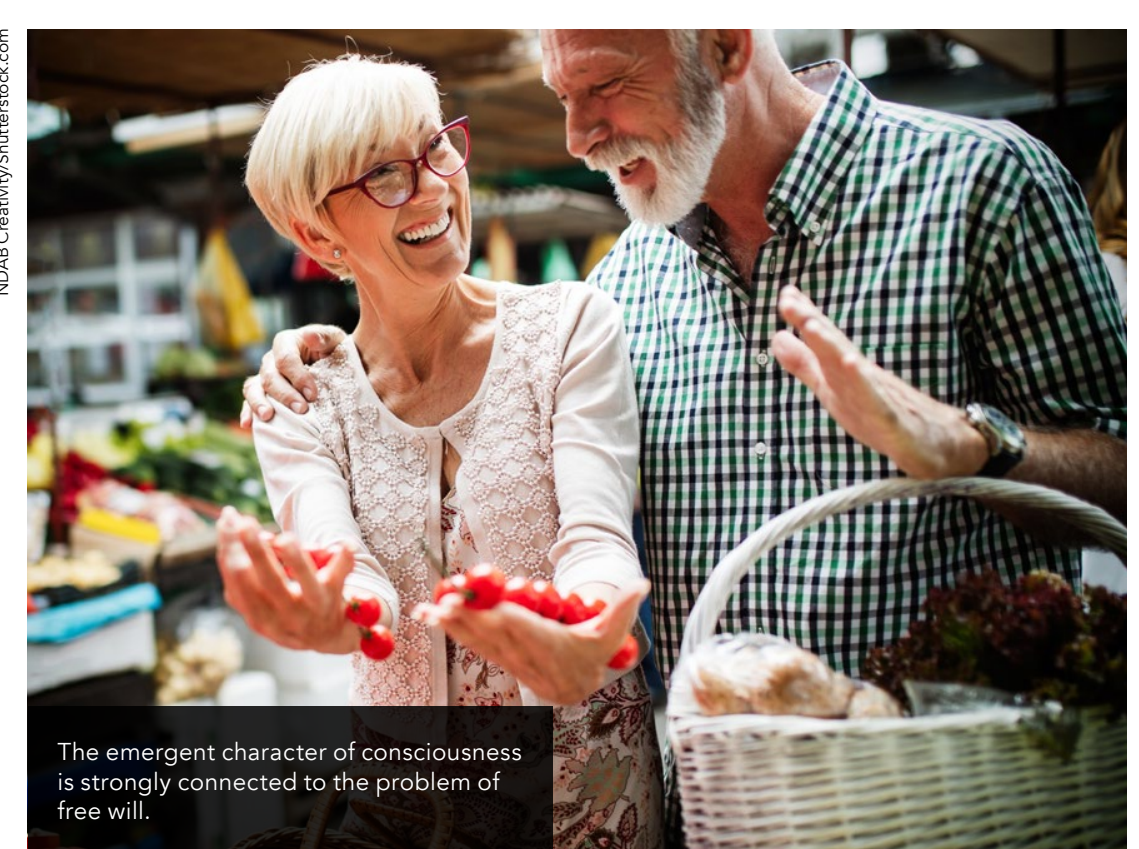

Subconsciousness is epistemologically emergent as its properties cannot be related to the properties of the low-level neurons. to subconsciousness. mind is epistemologically emergent, the mind-body problem cannot be subjective aspects of consciousness, (instances of subjective, conscions
experience), is not possible.

\section{CONSCIOUSNESS}

ONTOLOGICALLY EMERGENT

The ontologically emergent character

of consciousness is subsequently identified using a combinatorial analysis relating to the universal limits set by quantum mechanics. Professo Scheffel shows that the properties of consciousness are irreducible in principle to the lower level states and processes that form the basis of consciousness. He remarks that the emergent character of consciousness is strongly connected to the problem of free will.

FREE WILL

Professor Scheffel explains how

consciousness overlaps with the problem its behaviour could be simulated or predictable and therefore not free. If we

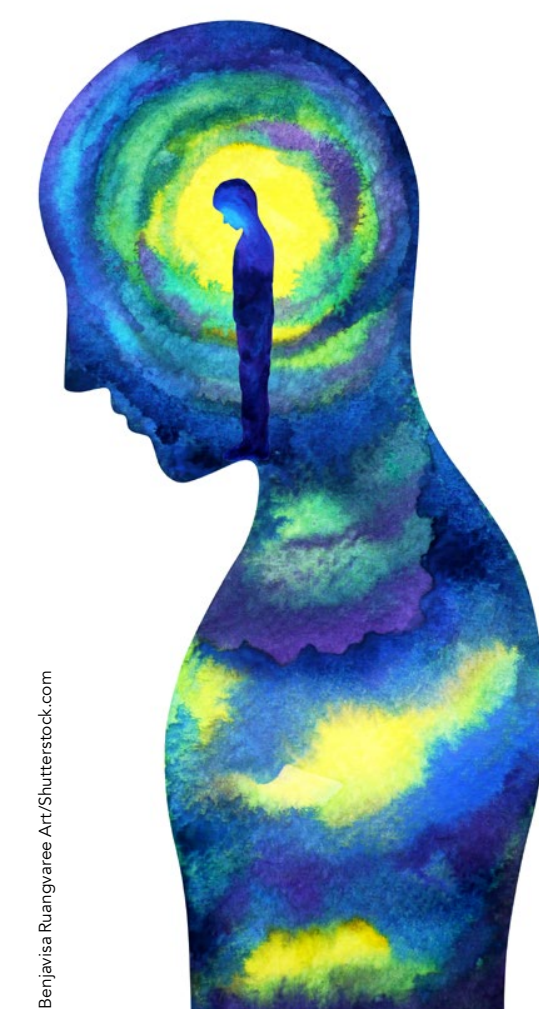

Having reached the conclusion that the were able to understand consciousness, fee will would be impossible.

The free will problem presumably is the most important existential problem of literature throughout history The common definition of free will as 'the ability to act differently' is part of the problem as it is difficult to apply scientific methods to establish whether we really can 'act differently'. How can, for example, a freely acting consciousness behave differently in identical situations?

\section{AN ONTOLOGICALLY}

OPEN SYSTEM

In order to make the concept of free will more suitable for scientific analysis, Professor Scheffel has refined the definition. "A conscious individual place according to its intentions, the intentions are no subconscios,y generated and if the individual's mind is an ontologically open system." An ontologically open system is a causa cannot be reduced to the states of its with a physicalist approach, Professor Scheffel finds that the ontologically emergent character of consciousness conscious agent, repeatedly placed in a particular situation, will identically epeat its behaviour, not due to standard downward causation Eve to emergent hus computer cannot be bult to explain this behaviour. He subsequently finds that the remaining requirements for free will are satisfied.

\section{CONCLUSION}

As a consequence of the emergent character of consciousness there is no possibility of reducing its characteristic properties to the low-level neurons on which it is based. Professor Scheffel concludes that from a non-reductive physicalist perspective, a solution to the mind-body problem, illuminating how mental properties can evolve from basic physical matter, is not possible. He then shows that free will is a consequence of fontological openness, a property that follows from the ontologically emergen renders it ontologically open. Thus, a

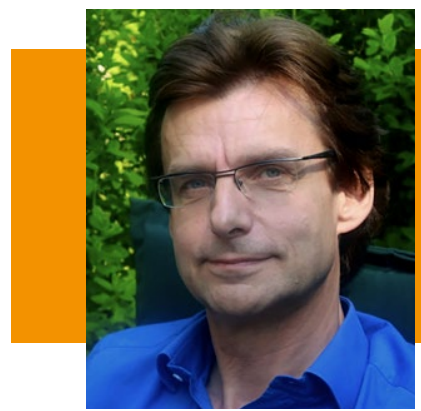

Behind the Research Professor Jan Scheffel

E: jans@kth.se T: $+467908939 \quad$ W: https://www.kth.se/profile/jans emergent in the epistemological sense.
Likewise, a similar argument is applied solved reductionistically. Moreover, a reductionistic understanding of the such as introspection and qualia of free will. If a reductionistic theory for high-level system with a future that associated low-level systems. Continuing

\section{Detail}

Jan Scheffel

Division of Fusion Plasma Physics

School of Electrical Engineering and Computer Science KTH Royal Institute of Technology

Bio

As Professor in theoretical fusion plasma physics at KTH, Jan Scheffel explores operational fits of and develops time-spectral numerical methods for mathematical modelling In analytical philosophy. his main areas of research are epistemology and philosophy of mind, in particular consciousness, free will and dualism.

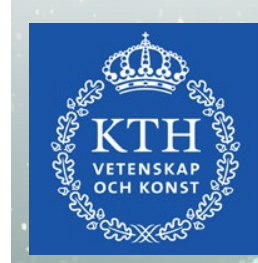

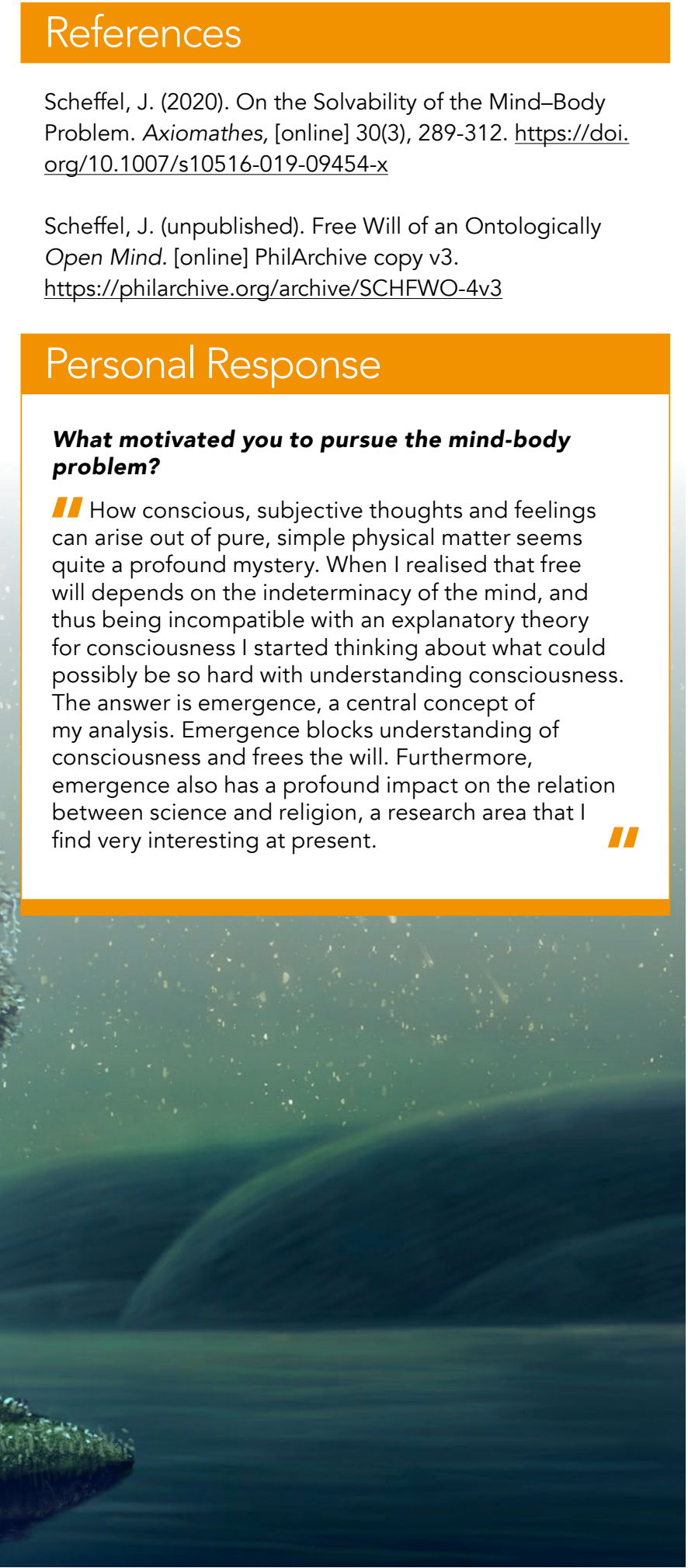

Scheffel, J. (2020). On the Solvability of the Mind-Body 30(3), 289-312. https.//doi.

Scheffel, J. (unpublished). Free Will of an Ontologically https://philarchive.org/archive/SCHFWO-4v3

How conscious, subjective thoughts and feeling quile a profound mystery. When Irealised tha free 政 y analysis. Emergence blocks understanding of ( religion, a research area that I

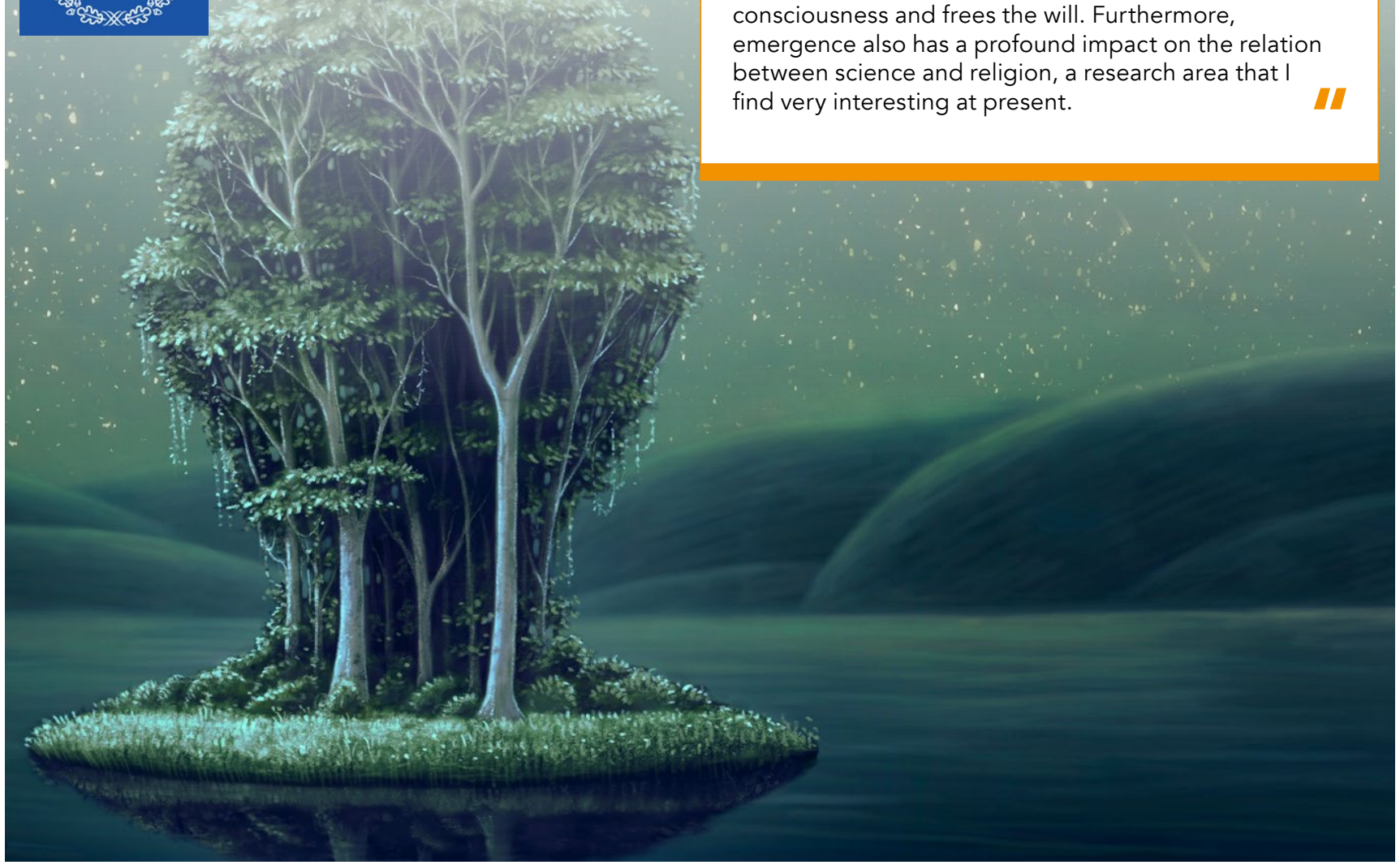

